\title{
Valor prognóstico do grau histológico (GH), grau nuclear (GN) e índice mitótico (IM) para pacientes com carcinoma da mama estádios II e III com linfonodos axilares comprometidos
}

\author{
Prognostic value of histological and nuclear grades and mitotic index for patients \\ with stages II and III breast carcinoma with positive axillary lymph nodes.
}

\author{
Luiz Augusto Freire Lopes', José Juvenal Linhares², Odair Ferraro ${ }^{3}$, Reginaldo Guedes Coelho Lopes', Fausto Farah Baracat ${ }^{5}$
}

\section{Resumo}

Objetivo: Avaliar o grau histológico (GH), grau nuclear (GN) e índice mitótico (IM) como fatores prognósticos para metástase em pacientes com carcinoma da mama, estádios clínicos II e III com linfonodos axilares comprometidos. Métodos: Cinqüenta e três pacientes foram submetidas a tratamento cirúrgico primário, através de mastectomia radical (Halsted) ou radical modificada (Patey), à quimioterapia e à radioterapia em parede torácica e fossa supraclavicular. O esquema quimioterápico compreendeu fluoracil, adriamicina e ciclofosfamida para pacientes com 50 anos ou menos; fluoracil, metrotrexate e ciclofosfamida para pacientes com mais de 50 anos. A presença de doença sistêmica foi, inicialmente, descartada e todas essas pacientes foram avaliadas por período mínimo de cinco anos ou até o aparecimento de metástases. O GH foi determinado através do método de Scarf-Bloom-Richardson modificado, o GN pelo método de Black modificado e o IM em dez campos de grande aumento (CGA) na periferia do tumor. Resultados: Das 53 pacientes, $27(50,9 \%)$ apresentaram metástases à distância. Destas, 18,5\% eram portadoras de tumores GH I (5/27); 14,8\% GH II (4/27) e 66,7\% GH III (18/27). Vinte seis $(49,1 \%)$ pacientes não apresentaram metástases. Destas, 38,4\% foram classificadas como grau I (10/ 26), $15,4 \%$ como grau II (4/26) e 46,2\% como grau III (12/26). O estudo do GN demonstrou que, das pacientes com metástases, 3,7\% (1/27) apresentavam tumor grau I, 22,2\% (6/27) tumores grau II e 74,1\% (20/27) tumores com grau III. Das sem metástases, 19,2\% (5/26) foram grau I, 34,6\% (9/26) grau II e 46,2\% (12/26) tumores grau III. Em relação ao IM, das pacientes com metástases, 14,8\% (4/27) apresentavam até cinco mitoses em 10 CGA, $25,9 \%$ (7/27) seis a dez mitoses e 59,3\% (16/27) mais de dez mitoses. Em relação às sem metástases, 38,5\% (10/26) apresentavam até cinco mitoses, 42,3\% (11/26) seis a dez mitoses e 19,2\% (5/26) mais de dez mitoses. Conclusōes: Os resultados demonstraram que o IM se associou com metástases à distância. Por outro lado, o GH e o GN não.

Palavras-chave: Neoplasias mamárias, Prognóstico, Índice mitótico.

Serviço de Ginecologia e Obstetrícia do Hospital do Servidor Público Estadual de São Paulo "Francisco Morato de Oliveira" (HSPE-FMO).

${ }^{1}$ Encarregado da Seção de Oncologia Pélvica do Serviço de Ginecologia e Obstetrícia do Hospital do Servidor Público Estadual de São Paulo "Francisco Morato de Oliveira" (HSPE-FMO).

${ }^{2}$ Professor Assistente de Ginecologia e Obstetrícia da Faculdade de Medicina - Campos de Sobral - da Universidade Federal de Ceará e Ex-Médico Ginecologista do Serviço de Ginecologia e Obstetrícia do Hospital do Servidor Público Estadual de São Paulo "Francisco Morato de Oliveira" (HSPEFMO).

${ }^{3}$ Encarregado da Seção de Patologia Mamária do Serviço de Ginecologia e Obstetrícia do Hospital do Servidor Público Estadual de São Paulo "Francisco Morato de Oliveira" (HSPE-FMO).

${ }^{4}$ Encarregado da Seção de Ginecologia do Serviço de Ginecologia e Obstetrícia do Hospital do Servidor Público Estadual de São Paulo "Francisco Morato de Oliveira" (HSPE-FMO).

${ }^{5}$ Diretor do Serviço de Ginecologia e Obstetrícia do Hospital do Servidor Público Estadual de São Paulo "Francisco Morato de Oliveira" (HSPE-FMO). Endereço para correspondência: José Juvenal Linhares, Rua Coronel Mont'Alverne, 1218. Bairro: Campo dos Velhos - Sobral-Ceará. CEP- 62100-000 E-mail: juvenallinhares@superig.com.br . 


\section{Abstract}

Objective: To evaluate the histological and nuclear grades and mitotic index for metastasis in patients with clinical stages II and III breast carcinoma with positive axillary lymph nodes. Methods: Fifty-three women were submitted to primary surgical treatment by radical (Halsted) or modified-radical (Patey) mastectomy and chemotherapy and radiotherapy in the chest wall and supraclavicular fossa. Chemotherapy included: fluorouracil, adriamycin, and cyclophosphamide for patients 50 years or younger and fluorouracil, methotrexate, and cyclophosphamide for patients over 50 years of age. Presence of systemic disease was ruled out initially and all patients were monitored for at least five years or until the appearance of metastasis. Histological grade was determined by the modified Scarf-Bloom-Richardson method, nuclear grade by the modified Black method, and mitotic index in X10 fields in the tumor periphery. Results: Of the 53 patients, 27 (50.9\%) presented distant metastases. Of these, 18.5\% (5/27) had histological grade I tumors, 14.8\% (4/27) histological grade II, and 66.7\% (18/27) grade III. Twenty-six patients (49.1\%) did not present metastases. Of these, 38.4\% (10/26) were classified as grade I, 15.4\% (4/26) grade II, and 46.2\% (12/26) grade III. Among patients with metastases, 3.7\% (1/27) presented nuclear grade I tumors, $22.2 \%(6 / 27)$ grade II, and 74.1\% (20/27) grade III. Of those without metastases, $19.2 \%(5 / 26)$ were nuclear grade I, 34.6\% (9/26) grade II, and 46.2\% (12/26) grade III. As for mitotic index, $14.8 \%$ (4/27) of patients with metastases had up to five mitoses per X10 field, 25.9\% (7/27) had six to ten mitoses, and 59.6\% (16/ 27) more than 10 mitoses. In patients without metastases, $38.5 \%$ (10/26) had up to five mitoses, $42.6 \%(11 / 26)$ six to ten mitoses, and 19.2\% (5/26) more than 10 mitoses. Conclusions: Mitotic index, but not histological grade or nuclear grade, was associated with distant metastases.

Key words: Breast cancer, Prognosis, Mitotic index.

\section{INTRODUÇÃO}

A experiência clínica demonstra que existem diferenças no comportamento biológico dos tumores da mama, no que diz respeito à agressividade e conseqüentemente ao prognóstico. Isso independe da terapêutica empregada e são ainda obscuros os mecanismos pelos quais esses fenômenos acontecem. Dessa forma, é muito importante entender e tentar explicar essa diversidade de comportamento para que se possam traçar estratégias de tratamentos diferentes para cada caso. Vários métodos permitem avaliar a agressividade dos tumores. Entre eles pode-se citar os indicadores genéticos, enzimáticos, de atividade proliferativa e morfológicos ${ }^{1,2}$.

Os indicadores morfológicos são os de menor custo e de mais fácil execução. Há muito tempo, sabe-se da importância do tamanho do tumor primário (T), comprometimento linfonodal axilar e perda da diferenciação celular, entre outros, como fatores relacionados ao intervalo livre de doença e à sobrevida global ${ }^{1,2}$.

O tamanho do tumor primário relaciona-se com a recidiva local e também com as recidivas à distância, revelando-se de grande importância prognóstica na evolução da doença . Alguns tumores, no entanto, apesar de muito pequenos, disseminamse rapidamente e são de péssimo prognóstico ${ }^{2}$.
Está estabelecido que a quantidade de linfonodos axilares comprometidos pela neoplasia é inversamente proporcional à sobrevida livre da doença e à sobrevida global ${ }^{3}$. Sabe-se, porém, que, entre as pacientes com axilas comprometidas, $40 \%$ sobrevivem por cinco anos e das pacientes com axilas livres, 20\% morrem nesse período 4 .

A característica histológica também reflete diferenças no grau de malignidade dos tumores 5 .

Greennhoug $^{6}$, Bloom ${ }^{7}$, Black et al. ${ }^{8}$ e Fisher et al. ${ }^{9}$ estudaram os aspectos morfológicos dos tumores da mama e concluíram haver boa correlação entre a diferenciação celular e a sobrevida. Fitzgibbons et al. ${ }^{33}$ e Hammond et al. ${ }^{34}$ inseriram o GH como categoria 1 dentre os parâmetros utilizados como fatores prognósticos para o câncer da mama.

O comportamento clínico dos tumores da mama é, muitas vezes, inesperado, não se entendendo exatamente os mecanismos que levam a resultados finais diferentes. Em virtude disso, surgiu o interesse em estudar a diferenciação celular nos tumores da mama, através do GH, GN e IM, para procurar buscar associações entre esses fatores com metástases à distância em cinco anos, a fim de determinar qual desses indicadores melhor se associariam com esses eventos em nossa casuística. 


\section{OBJETIVOS}

Verificar o valor prognóstico do GH, GN e do IM para metástases em pacientes portadoras de carcinoma da mama nos estádios clínicos II e III, com linfonodos axilares comprometidos.

\section{CASUÍSTICA E MÉTODOS}

Este estudo refere-se a um grupo de 53 mulheres atendidas no Setor de Mastologia do Serviço de Ginecologia e Obstetrícia do Hospital do Servidor Público Estadual "Francisco Morato de Oliveira" - São Paulo, no período de 1982 a 1987.

Todas eram portadoras de carcinoma da mama do tipo histológico ductal invasivo, com linfonodos axilares comprometidos pela neoplasia, inseridos, pois, nos estádios II e III.

O exame histológico do tumor inicial foi refeito para todas as pacientes, sobre lâminas arquivadas, com cortes de 4 micras de espessura e coradas pela técnica de hematoxilina-eosina ou em novas lâminas confeccionadas em blocos de parafina também arquivados.

A leitura foi realizada sempre em sentido horário e os tumores foram classificados quanto aos seguintes critérios: GH, GN e IM.

O GH foi determinado através do método de ScarfBloom-Richardson (SBR), modificado de acordo com Elston e Ellis5. O GN foi determinado pelo método de Black, modificado segundo Fisher et al. .

O IM foi avaliado na periferia do tumor. Contaramse as mitoses em dez campos de grande aumento - CGA (x 40) com um microscópio da marca Nikkon - Labophot, com diâmetro de campo de $0,44 \mathrm{~mm}$ e área do campo de $0,152 \mathrm{~mm}$. Conferiu-se um ponto aos tumores com zero a cinco figuras de mitoses por dez CGA, dois pontos àqueles com seis a dez e três pontos quando mais de dez foram observadas, segundo Elston e Ellis ${ }^{5}$. Não foram consideradas as figuras que poderiam ser confundidas com núcleos picnóticos e com cariorréxis.

A distribuição das pacientes por faixa etária está ilustrada na tabela 1 . A idade média das pacientes foi de 51,5 anos, variando entre 31 e 77 anos.

Tabela 1. Faixa etária das pacientes

\begin{tabular}{lcc}
\hline IDADE & N & $\%$ \\
\hline$\leq 35$ anos & 3 & 5,7 \\
36 a 50 anos & 22 & 41,5 \\
51 a 70 anos & 26 & 49,1 \\
$\geq 71$ anos & 2 & 3,7 \\
\hline TOTAL & 53 & 100 \\
\hline
\end{tabular}

$\chi^{2}=0,03$

\section{TAMANHO DA AMOSTRA:}

O tamanho da amostra foi calculado pelo software Epi-info versão 6.0, antes do início do estudo, fixando-se um erro $\alpha$ de $5 \%$, com intervalo de confiança de $95 \%$.

Apesar de estudos anteriores demonstrarem recidivas, em quatro anos variando entre 43 e $70 \%$, em pacientes que apresentavam tumor da mama com axila comprometida, uma análise mais realista da incidência de metástases neste grupo demonstrou uma variação entre 35 e $51 \%$ em cinco anos ${ }^{5}$. Desta forma, determinou-se um tamanho mínimo necessário para representatividade da amostra de 22 pacientes com metástases em cinco anos.

\section{Seleção dos PaCIenteS}

Todas as pacientes foram submetidas a tratamento cirúrgico primário através da mastectomia radical, segundo Halsted ou da mastectomia radical modificada, segundo Patey.

Como tratamento adjuvante, foram submetidas à radioterapia e à quimioterapia. A radioterapia foi realizada em parede torácica e fossa supraclavicular. $\mathrm{O}$ esquema quimioterápico utilizado foi fluoracil, adriamicina e ciclofosfamida (FAC) para as pacientes com idade igual ou inferior a 50 anos (25 pacientes) e ciclofosfamida, methotrexate e fluoracil (CMF) para aquelas com idade superior a 50 anos (28 pacientes).

\section{CRITÉRIOS DE INCLUSÃo}

Pacientes com carcinoma da mama: tipo histológico ductal invasivo, classificado como T2 e T3, com axilas comprometidas (Classificação TNM, UICC, 2004) e ausência de metástases. A presença de metástases foi descartada por meio do exame clínico, da radiografia de tórax, da cintilografia óssea, da ultra-sonografia abdominal e das provas de função hepática.

\section{Seguimento}

As pacientes foram acompanhadas por um período mínimo de cinco anos ou até o aparecimento de metástases. Este diagnóstico foi feito por meio da clínica e de métodos de imagem (radiografia, ultra-sonografia, cintilografia, tomografia computadorizada).

\section{ANÁLISE ESTATÍSTICA}

A análise estatística dos resultados foi realizada com o teste do qui-quadrado a fim de se detectar diferenças significativas entre os casos sem e com metástases, de acordo com a faixa etária das pacientes, o grau histológico, o grau nuclear e o índice mitótico.O nível de significância $\alpha$ foi de $5 \%$. 


\section{RESULTADOS}

Conforme ilustrado na tabela 2 , das 53 pacientes pertencentes a este estudo, 27 (51\%) apresentaram metástases à distância no período de cinco anos.

Ao se comparar a idade das pacientes entre o grupo com e sem metástases, conforme ilustra a tabela 2 , não houve diferença estatisticamente significativa com $\chi^{2}=0,16$ e $\mathrm{p}=0,68$. Entre as pacientes que apresentaram metástases, 12 tinham idade igual ou inferior a 50 anos $(44,5 \%)$ e 15 idade superior a 50 anos $(55,5 \%)$. Entre as que não apresentaram recidivas, 13 tinham idade igual ou inferior a 50 anos (50\%) e 13 mais de $50(50 \%)$ (Tabela 2).

Tabela 2. Mulheres com carcinoma da mama e axila comprometida segundo a idade e a detecção de metástases em cinco anos

\begin{tabular}{ccccc}
\hline \multirow{2}{*}{ IDADE (anos) } & \multicolumn{4}{c}{ METÁSTASES } \\
\cline { 2 - 5 } & \multicolumn{3}{c}{ SIM } & \multicolumn{3}{c}{ NÃO } \\
\hline & $\mathrm{N}$ & $\%$ & $\mathrm{~N}$ & $\%$ \\
$\leq 50$ & 12 & 44,5 & 13 & 50,0 \\
$\geq 51$ & 15 & 55,5 & 13 & 50,0 \\
\hline TOTAL & 27 & 100 & 26 & 100 \\
\hline
\end{tabular}

$\chi^{2}=0,16$ e $\mathrm{p}=0,68$

Quanto à diferenciação dos tumores pelo $\mathrm{GH}, 15$ $(28,3 \%)$ eram grau I, oito $(15,09 \%)$ grau II e $30(56,6 \%)$ grau III (Tabela 3). Das 27 pacientes com metástases, $18,5 \%$ apresentavam tumores de grau histológico I (5/ 27), $14,8 \%$ grau II (4/27) e 66,7\% grau III (18/27). Dentre aquelas sem metástases (26), 38,4\% (10/26) eram portadoras de tumores grau I, 15,4\% (4/26) grau II e $46,2 \%(12 / 26)$ tinham tumores grau III (Tabela 3). A análise dos resultados não demonstrou associação entre o grau histológico e o aparecimento de metástases em cinco anos, $\chi^{2}=2,85$ e $\mathrm{p}=0,24$ (Tabela 3 ).

Tabela 3. Pacientes com câncer da mama segundo o grau histológico e detecção de metástases no período de cinco anos

\begin{tabular}{ccccc}
\hline GRAU & \multicolumn{4}{c}{ METÁSTASES } \\
\cline { 2 - 5 } HISTOLÓGICO & \multicolumn{3}{c}{ SIM } & NÃO \\
\hline & N & $\%$ & N & $\%$ \\
I & 05 & 18,5 & 10 & 38,4 \\
II & 04 & 14,8 & 04 & 15,4 \\
III & 18 & 66,7 & 12 & 46,2 \\
\hline TOTAL & 27 & 100,0 & 26 & 100,0 \\
\hline
\end{tabular}

$\chi^{2}=2,85$ e $\mathrm{p}=0,24$

Quanto ao GN, constatou-se que, das 53 pacientes estudadas, seis $(11,3 \%)$ tinham tumores classificados como GN I, $15(28,30 \%)$ eram portadoras de tumores
GN II e 32 (60,3\%) tinham tumores GN III (Tabela 4). Ao se avaliar o GN dos tumores, observou-se que, das 27 das pacientes com metástases, 3,7\% (1/27) apresentavam tumor com GN I, 22,2\% (6/27) com GN II e $74,1 \%(20 / 27)$ com GN III. Entre as que não apresentaram metástases, $19,2 \%(5 / 26)$ tinham tumores com GN I, 34,6\% (9/26) com GN II e 46,2\% (12/26) com GN III, como ilustra a tabela 4 . A análise estatística dos resultados também não revelou correlação entre o $\mathrm{GN}$ e a recidiva à distância em cinco anos, $\operatorname{com} \chi^{2}=5,25$ e $\mathrm{p}=0,07$ (Tabela 4).

Tabela 4. Pacientes com câncer da mama segundo o grau nuclear e detecção de metástases no período de cinco anos

\begin{tabular}{ccccc}
\hline \multirow{2}{*}{ GRAU NUCLEAR } & \multicolumn{4}{c}{ METÁSTASE } \\
\cline { 2 - 5 } & \multicolumn{3}{c}{ SIM NÃO } \\
\hline & $\mathrm{N}$ & $\%$ & $\mathrm{~N}$ & $\%$ \\
II & 01 & 3,7 & 05 & 19,2 \\
III & 06 & 22,2 & 09 & 34,6 \\
\hline TOTAL & 20 & 74,1 & 12 & 46,2 \\
\hline
\end{tabular}

$\chi^{2}=5,25$ e $\mathrm{p}=0,07$

Em relação ao IM, das 53 pacientes estudadas, 14 $(26,4 \%)$ apresentavam tumores com 0 a 5 figuras de mitoses em dez CGA, 18 (34\%) eram portadoras de tumores com seis a dez mitoses e 21 pacientes $(39,6 \%)$ apresentavam tumores com mais de dez mitoses (Tabela 5). Das pacientes com metástases em cinco anos, $14,8 \%$ (4/27) apresentavam tumores com até cinco figuras de mitoses, $25,9 \%$ (7/27) com seis a dez mitoses e 59,2\% (16/27) com mais de dez mitoses em dez CGA (Tabela 5). Em relação às 26 pacientes sem metástases, 38,5\% (10/ 26) tinham tumores com até cinco figuras de mitoses, $42,3 \%(11 / 26)$ apresentavam neoplasias com seis e dez figuras de mitoses e 19,2\% (5/26) mais de dez figuras de mitoses (Tabela 5). A análise estatística dos resultados revelou que o IM apresentou relação positiva com a recidiva tumoral à distância em cinco anos, com a contagem mitótica maior do que dez associada à presença de metástases $\left(\chi^{2}=9,21, p=0,01\right)$.

Tabela 5. Pacientes com câncer da mama segundo o índice mitótico e detecção de metástases no período de cinco anos

\begin{tabular}{ccccc}
\hline ÍNDICE & \multicolumn{4}{c}{ METÁSTASES } \\
\cline { 2 - 5 } MITÓTICO & \multicolumn{3}{c}{ SIM } & \multicolumn{2}{c}{ NÃO } \\
\hline & $\mathrm{N}$ & $\%$ & $\mathrm{~N}$ & $\%$ \\
0 a 5 & 04 & 14,8 & 10 & 38,5 \\
6 a 10 & 07 & 25,9 & 11 & 42,3 \\
$>10$ & 16 & 59,3 & 05 & 19,2 \\
\hline TOTAL & 27 & 100,0 & 26 & 100,0 \\
\hline
\end{tabular}

$\chi^{2}=9,21$ e $\mathrm{p}=0,01$ 


\section{DISCUSSÃO}

Os tumores da mama têm um comportamento biológico muitas vezes inesperado. Algumas pacientes não tratadas apresentam sobrevida prolongada semelhante à daquelas no mesmo estádio clínico e submetidas a vários esquemas terapêuticos ${ }^{10}$. Vermund et al. ${ }^{11}$, em 1308 pacientes não tratadas, encontraram índice de sobrevivência de 19\% em cinco anos e de 5\% em dez anos. Bloom ${ }^{12}$, em revisão de 1728 mulheres com câncer da mama não tratadas, encontrou índice de sobrevivência média de 39,9 meses.

Constatou-se que a cirurgia utilizada no tratamento primário mostrou recidiva da enfermidade em $20 \%$ das pacientes sem comprometimento dos linfonodos axilares e em $70 \%$ daquelas com linfonodos comprometidos, no período de cinco $\operatorname{anos}^{13}$. Isso evidenciou a necessidade do controle da doença subclínica para se obter resultados melhores no tratamento das pacientes com essa neoplasia. Por outro lado, o tratamento adjuvante, apesar da evolução nas últimas décadas, é efetivo apenas para algumas pacientes ${ }^{10}$. De maneira geral, pacientes com tumores da mama no estádio clínico II apresentam índice de mortalidade de $36 \%$ nos primeiros cinco anos. Naquelas submetidas à poliquimioterapia, ocorre redução de $25 \%$ na mortalidade, que cairia, portanto, para $27 \%$. Em resumo, de 100 pacientes com câncer da mama no estádio clínico II e submetidas à poliquimioterapia, nove estariam curadas e 27 teriam aumento de dezoito a vinte e quatro meses na sobrevida. Portanto, dessas 100 pacientes tratadas, 36 obteriam algum benefício. As outras 64 submetidas à quimioterapia seriam, estatisticamente, medicadas desnecessariamente ${ }^{14}$.

A idade constitui um fator associado, relevante no que diz respeito à evolução das pacientes. Aquelas na prémenopausa apresentam neoplasias com indicadores patológicos que caracterizariam os casos de comportamento biológico mais agressivo e consequentemente de pior prognóstico ${ }^{15}$.

Guerrero et al. ${ }^{16}$, em estudo de 560 pacientes com câncer da mama, encontraram maior incidência de comprometimento dos linfonodos axilares naquelas com idade inferior a 50 anos. No presente estudo, os dois grupos, com idade igual ou inferior a 50 anos e superior a 50, não apresentaram diferença quanto à recidiva à distância, o que está de acordo com os trabalhos de Mueller et al. ${ }^{17}$ e Singletary et al. ${ }^{18}$.

Os aspectos morfológicos microscópicos podem estar relacionados com a evolução dos tumores. Assim, está relatado, desde 1925, que a diferenciação celular se associa com a sua evolução ${ }^{19}$. Greenhoug mostrou evolução pior para os tumores com $\mathrm{GH}$ mais elevado, isto é, tumores considerados menos diferenciados. Apesar da boa correlação prognóstica do GH, 10 a 15\% das pacientes com tumores invasivos da mama, GH I, apresentam metástases quando acompanhadas por períodos prolongados ${ }^{20}$. Além disso, não parece haver grande influência do GH na previsão da disseminação nos tumores de grande volume ${ }^{21}$. O grande desafio dos pesquisadores, atualmente, é identificar novos fatores prognósticos ainda não conhecidos.

Fitzgibbons et al. ${ }^{33}$ e Hammond et al. ${ }^{34}$ inseriram o GH como categoria 1 dentre os parâmetros utilizados como fatores prognósticos para o câncer da mama. No entanto, Younes et al..$^{22}$ encontraram falta de significado prognóstico do $\mathrm{GH}$ em pacientes com tumores da mama e axila negativa. Ladekarl ${ }^{23}$ afirma que a baixa reprodutibilidade reduz o valor clínico da graduação morfológica dos tumores. Neste trabalho, não foi encontrada uma diferença significativa entre GH e recidiva à distância em cinco anos de evolução, de acordo, pois, com esses últimos autores citados. É provável que, com o aumento do número de casos, pudesse ocorrer esta associação, como encontrado em trabalhos consagrados na literatura citados anteriormente.

O GN, apresenta por si, sem a associação com o grau de formação tubular, boa correlação com a sobrevida geral e livre de doença ${ }^{8,26}$. De forma diversa do $\mathrm{GH}$, esse pode ser aplicado a todos os tipos histológicos de carcinoma ${ }^{24,25,26}$. Martinez-Arribas et al. ${ }^{35}$ mostraram que pacientes com tumores GN III apresentaram uma associação importante com outros parâmetros relacionados ao mau prognóstico de pacientes com câncer da mama (ex: alta expressão de $\mathrm{Ki}^{67}$, ausência de receptores de estrogênio e progesterona e alta expressão de Cerb-B2), favorecendo assim uma correlação positiva entre esse achado histológico e uma maior possibilidade de metástases. Neste trabalho, também não foi encontrada uma relação entre GN e recidiva à distância em cinco anos. É provável que, com o aumento do número de casos, pudesse ocorrer esta associação, como encontrado na literatura.

O IM constituiu-se inicialmente no único meio de avaliação da cinética celular e foi bem estabelecido como fator prognóstico nos tumores mesenquimais, inclusive nos da mama ${ }^{27}$. A determinação precisa do número de mitoses é difícil porque em alguns tumores a sua ocorrência é escassa, e necessitam ser avaliadas até 10.000 células para se encontrar cinco ou mais figuras de mitose. Essas mitoses devem ser distinguidas dos núcleos picnóticos e com cariorréxis, o que torna trabalhosa a sua determinação, principalmente nos tumores com baixo número de mitoses. O IM está diretamente relacionado com alguns fatores como a proporção de células que está no ciclo celular, com a 
duração do mesmo (tempo entre duas mitoses) e com o tempo requerido para completá-las. Esse último, embora seja o mais breve do ciclo celular, pode variar de quatro a cinco vezes entre alguns tumores. A duração prolongada da mitose, em alguns deles, produz um alto IM sem refletir necessariamente a taxa real de proliferação celular. Apesar disso, existe boa correlação entre a determinação do componente proliferativo por timidina tritiada e fração da fase $S$ por citometria de fluxo, quando comparadas com o IM.

Baak et al. ${ }^{28}$, Van Diestet et al. ${ }^{29}$, Caly et al. ${ }^{30}$ constataram que o IM determinado em dez campos de grande aumento (CGA), na área mais rica de mitoses do tumor, representou fator prognóstico comparável ao comprometimento dos linfonodos axilares e melhor do que o tamanho do tumor, $\mathrm{GH}$ ou variaçôes nucleares. Van Diest et al. ${ }^{31}$ consideraram o IM o fator proliferativo com maiores índices de reprodutibilidade e o fator prognóstico independente de maior valor. Medri et al. ${ }^{32}$ observaram que o grande número de figuras de mitoses estavam associadas com maior probabilidade de metástases à distância em pacientes com linfonodos negativos.

A contagem mitótica aparentemente é preditiva do curso das neoplasias epiteliais malignas da mama e é um método de determinação da fração proliferativa dos tumores. Não exige procedimentos especiais, é de baixo custo e fácil execução e pode ser aplicado em estudos retrospectivos. Sua sensibilidade na determinação da fração proliferativa parece ser menor do que em métodos mais complexos de análises da fração do DNA, porém não se sabe se é menos efetivo como valor preditivo de sobrevida.

Este fator prognóstico foi o único, no presente trabalho, que mostrou relação positiva com a recidiva à distância em cinco anos. Nesse caso, a contagem mitótica maior do que dez associou-se com a presença de metástases, o que está de acordo com a literatura.

Logo, concluiu-se que o grau histológico e o grau nuclear não apresentaram correlação positiva quanto às recidivas à distância em cinco anos em tumores da mama estádios clínicos II e III com linfonodos comprometidos. O índice mitótico foi o único fator prognóstico, no presente trabalho, que mostrou relação positiva com recidiva à distância em cinco anos, em tumores da mama estádios clínicos II e III com linfonodos comprometidos.

\section{REFERÊNCIAS BIBLIOGRÁFICAS}

1. Carter CL, Allen C, Henson DE. Relation of tumor size, lymph node status and survival in 24.740 breast cancer cases. Cancer. 1989;63:181.

2. Leis HP Jr. Parametros pronosticos para el cancer de mama. In : Bland KI, Copeland III EM, editores. La mama: manejo multidiciplinario de las enfermedades benignas y malignas. Buenos Aires: Editorial Medica Panamericana; 1993. p.427.
3. Fisher B, Slack NH, Bross ID. Cancer of the breast: size of neoplasm and prognosis. Cancer. 1969;24:1071-80.

4. Black MM, Speer FD, Opler SR. Structural representations of tumor-host relationship in mammary carcinoma: biologic and prognostic significance. Am J Clin Pathol. 1956;26:250-265.

5. Elston CW, Ellis IO. Pathological prognostic factors in breast cancer. The value of histological grade in breast cancer: experience from a large study with long term follow up. Histopathology. 1991;19:403-10.

6. Greenhoug RB. Varying degrees of malignancy in cancer of the breast. J Cancer Res. 1925;9:452-63.

7. Bloom HJG. Prognosis in carcinoma of the breast. Br J Cancer. 1950;4:259-88.

8. Black MM, Opler SR, Speer FD. Survival in breast cancer cases in relation to the structure of the primary tumor and regional lymph nodes. Surg Gynecol Obstet. 1955;100:543.

9. Fisher ER, Redmond C, Fisher B. Histologic grading of breast cancer. Pathol Annu. 1980;15:239-51.

10. Dhingra K, Hortobagyi GN. Critical evaluation of prognostic factors. Semin Oncol. 1996;23:436-45.

11. Vermund L. Trends in radiotherapy of breast cancer. Proceedings of the Fifth National Cancer Conference; 1964 Sep; Philadelphia, US. Philadelphia: JB Lippincott; 1964.

12. Bloom HJG. Survival of woman with untreated breast cancer: past and present. In: Forrest APM, KlumKler PB, editors. Prognostic factors in breast cancer. Baltimore: Williams e Wilkins; 1968. p.3.

13. Fisher B, Ravdin RG, Ausman RK, Slack NH, Moore GE, Noer RJ. Surgical adjuvant chemotherapy in cancer of the breast results of a decade of cooperative investigation. Ann Surg. 1968;168:337-56.

14. O'Grady LF, Lindfors KK, Howell LP, Rippon MB. A practical approach to breast disease. Boston: Little Brown and Company; 1995. p. 217-20.

15. Winchester DP. Breast cancer in young women. Surg Clin North Am. 1996;76:279-87.

16. Guerrero RJM, Hernandez HM, Migallon JAS, Guerreiro ES. Estudio retrospectivo (1982-1986) de los fatores prognosticos em 500 casos de cancer de mama. Rev Inst Nat Cancerol (Mex). 1994;40:71-5.

17. Mueller CB, Ames F, Anderson GD. Breast cancer in 3.558 woman: age as a significant determinant in the rate of dying and causes of death. Surgery. 1978;83:123-32.

18. Singletary SE, Shallenberger R, Guinee VF: Breast cancer in the elderly. Ann Surg. 1993;218:667-71.

19. Greenhoug RB. Varying degrees of malignancy in cancer of the breast. J Cancer Res. 1925;9:452-63.

20. Leong AC, Hanby AM, Potts HW, Tan DS, Skilton D, Ryder K, et al. Cell cycle proteins do not predict outcome in grade I Infiltrating ductal carcinoma of the breast. Int J Cancer. 2000;89:26-31. 
21. Chagas CR. Câncer de mama: iniciação e crescimento tumoral, formação de metástases, aspectos de interesse clínico. Femina. 1996;24:11-24.

22. Younes M, Laucirica R. Lack of prognostic significance of histological grade in node-negative invasive breast carcinoma. Clin Cancer Res. 1997;3:601-4.

23. Ladekarl M. Objective malignancy grading: a review emphasizing unbiased stereology applied to breast tumors. APMIS.1998;(79 suppl):1-34.

24. Simpson JF, Page DL. The role of pathology in the premalignancy and as a guide for treatment and prognosis in breast cancer. Semin Oncol. 1996; 23:428-35.

25. Carvalho FM. Histopatologia dos carcinomas de mama. In: Piato S, editor. Mastologia. São Paulo: Roca; 1995. p.139-54.

26. Fisher ER, Redmond C, Fisher B, Bass G. Pathologic findings from the National Surgical Adjuvant Breast and Bowel Projects ( NSABP ). Prognostic discriminants for 8year survival for node-negative invasive breast cancer patients. Cancer. 1990;65(9 suppl):2121-8.

27. Norris HJ, Taylor HB. Relationship of histological features to behavior of cystosarcoma phyllodes: analysis of ninetyfour cases. Cancer. 1967;20:2090.

28. Baak JP, Van Dop H, Kurver PH, Hermans J. The value of morphometry to classic prognostic factors in breast cancer. Cancer. 1985;56:374-82.

29. Van Diest PJ, Baak JP, Matze-Cok P, Wisse-Brekelmans EC, Van Galen CM, Kurver PH, et al. Reproducibility of mitoses counting in 2469 breast cancer specimens : results from the Multicenter Morphometric Mammary Carcinoma
Project. Hum Pathol. 1992;23:603-7.

30. Caly M, Genin P, Ghuzlan AA, Elie C, Freneaux P, Klijanienko J, et al. Analysis of correlation between mitotic index, MIB1 score and S-phase fraction as proliferation markers in invasive breast carcinoma: methodological aspects and prognostic value in a series of 257 cases. Anticancer Res. 2004;24(5B):3283-8.

31. van Diest PJ, van der Wall E, Baak JP. Prognostic value of proliferation in invasive breast cancer: a review. J Clin Pathol. 2004;57(7):675-81.

32. Medri L, Volpi A, Nanni O, Vecci AM, Mangia A, Schittulli $F$, et al. Prognostic relevance of mitotic activity in patients with node-negative breast cancer. Mod Pathol. 2004;17(8):1024.

33. Fitzgibbons PL, Page DL, Weaver D, Thor AD, Allred DC, Clark GM, et al. Prognostic factors in breast cancer: College of American Pathologists Consensus Statement 1999. Arch Pathol Lab Med. 2000 Jul;124(7):966-78.

34. Hammond ME, Fitzgibbons PL, Compton CC, Grignon DJ, Page DL, Fielding LP, et al. College of American Pathologists Conference XXXV: solid tumor prognostic factors-which, how and so what? Summary document and recommendations for implementation. Cancer Committee and Conference Participants. Arch Pathol Lab Med.2000 Jul;124(7):958-65.

35. Martinez-Arribas F, Martin-Garabato E, Lafuente P, Tejerina A, Lucas R, Sanchez J, et al. Proliferation measurement in breast cancer by two different methods. Anticancer Res. 2006 Jan-Feb;26(1A):199-202. 